\section{IMPLANTING THE RIGHT KNOWLEDGE}

It is not a secret that patient expectations for dental implant therapy are getting higher. The challenges in implant dentistry have shifted from osseointegration to the pink and white parts of dental implants. Dentists in the United Kingdom have a great opportunity to gain sound grounding in aesthetic implant dentistry by joining the one year part time 'Evidence Based Et Clinical Course in Implant Dentistry' by The British Academy of Implant \& Restorative Dentistry (BAIRD Academy) which is run under the direction of Professor Marco Esposito. Marco is the Editor-in-Chief of the European Journal of Oral Implantology in addition to a group of prominent national and international speakers and mentors.

This private comprehensive course is designed to provide dentists with the highest level of knowledge and skills related to the placement and restoration of dental implants. Dentists will learn the biologic and clinical principles that are the basis of the discipline. This evidencebased course provides a rich clinical experience that will allow participants to assume a leadership role within the world of dental implantology.

Practitioners are clinically trained via basic and advanced didactic evidence-based seminars, handson workshops and clinical sessions (on patients provided by the course). All topics pertinent to surgical and prosthetic dental implantology are covered: eg diagnosis and treatment planning; surgical protocols (3D implant placement, sinus grafting, guided bone regeneration, soft tissue grafting, prosthetic protocols); implant and abutment level procedures; screw $v s$. cement retained; prosthesis design; fixed $v s$. overdentures; and aftercare. Clinical procedures include all of the aforementioned protocols performed on course patients under the supervision of experienced mentors at the faculty of BAIRD Academy.

This Leeds-based, one-year, part-time course is running for the sixth year with very good feedback from previous graduates. The course is beginning on 25th September 2015 and continues until the end of May. The course comprises of seven modules, each of them delivered over a long weekend once a month, followed by a case presentation and theory exams in June 2016. Upon completing the course and successfully presenting the training portfolio, course participants will be awarded a Certificate of Evidence Based \&t Clinical Implant Dentistry. Successful candidates will also have the opportunity to accredit their training towards the Post Graduate University Implant Diploma at San Raffaele University in Milan.

For more information and course application form, visit www.baird.uk.com or contact us at admin@baird.uk.com.

\title{
BEWARE OF LIABILITY FOR UNDERPERFORMANCE WHEN BUYING A DENTAL PRACTICE
}

The specialist dental team at Hempsons can help you to buy or sell your dental practice, and they are very well aware of the particular issues which affect dentists. For example, if you are planning to take over

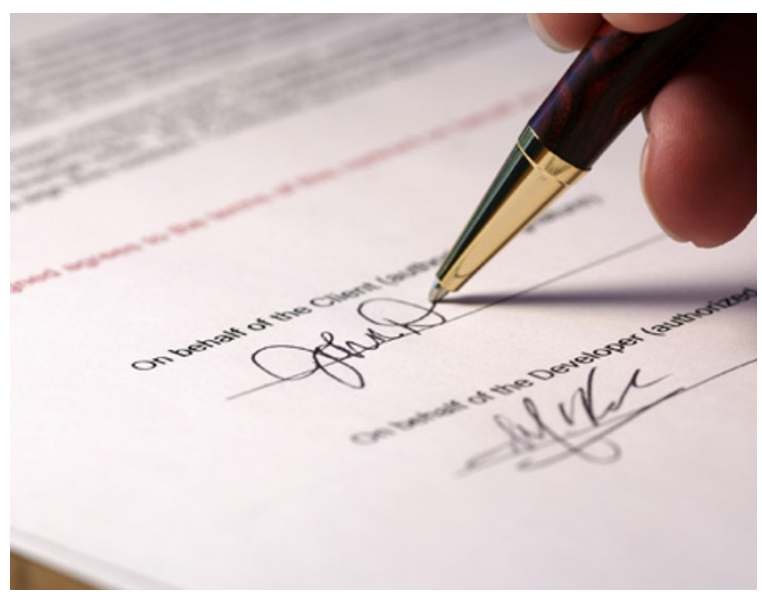

an NHS contract when you buy a practice, it is very important that the contract includes provision for any liability for underperformance prior to your purchase of the practice, even if you are not aware of any underperformance at the time of the purchase. Otherwise, you could be liable for that underperformance, once you have become the provider/contractor. This may lead to you having monies clawedback from your monthly payments from the BSA, with no recourse to the vendor.

For more information, call Faisal Dhalla on 01423724019 or email f.dhalla@hempsons.co.uk.

\section{JOIN THE CHALLENGE}

Philips is recruiting dental professionals to take part in a study it is running in the autumn to assess the effectiveness the Sonicare toothbrush. All GDC registered dentists, hygienists and therapists can sign up to take part in the Sonicare Challenge and will receive a Sonicare EasyClean toothbrush fitted with an InterCare brush head, to experience Sonicare cleaning for themselves. In return all they need to do is try the brush for 28 days and let Phillips have their feedback.

The patented technology of Sonicare uses a sonic cleaning action, which combines high frequency (number of brush strokes) and amplitude (distance of bristle movements) for a unique clean. Challenge participants will find that unlike other electric brushes they may have used, this sonic action agitates the toothpaste and fluid in the mouth creating a dynamic fluid cleaning to clean between teeth and along the gum line where bristles fail to reach. Builtin technological innovation also prevents overbrushing which can lead to receding gums and tooth sensitivity.

The Sonicare EasyClean being used in the trial will be coupled with an InterCare brush head which features extra-long filaments to reach deep between teeth and remove six times the amount of plaque biofilm a manual toothbrush can achieve." The brush head is also available in two sizes to accommodate mouth size and site specific requirements.

The evaluation of the Sonicare brush will run from August 2015 until stocks last. Dental professionals will be provided with the Sonicare and to thank them for participating will be allowed to keep it at the end of the 30 day trial period after reporting their findings.

To sign up to take part visit www.philips-tsp.co.uk/trial19.

* Compared with a DiamondClean toothbrush with an InterCare brush head. 